13,05

\title{
Наноструктуры ферромагнитный металл/полупроводник на основе силицидов железа
}

\author{
(C) С.Г. Овчинников ${ }^{1-3}$, С.Н. Варнаков ${ }^{1,2}$, С.А. Лященко ${ }^{2}$, И.А. Тарасов ${ }^{1,2}$, И.А. Яковлев ${ }^{2}$, Е.А. Попов ${ }^{2}$, \\ С.М. Жарков ${ }^{1}$, Д.А. Великанов ${ }^{1}$, А.С. Тарасов ${ }^{1}$, В.С. Жандун ${ }^{1,2}$, Н.Г. Замкова ${ }^{1}$ \\ ${ }^{1}$ Институт фризики им. Л.В. Киренского СО РАН, \\ Красноярск, Россия \\ ${ }^{2}$ Сибирский государственный аэрокосмический университет им. акад. М.Ф. Решетнева, \\ Красноярск, Россия \\ ${ }^{3}$ Национальный исследовательский ядерный университет „МИФИ“, \\ Москва, Россия \\ E-mail: sgo@iph.krasn.ru
}

Ферромагнитные монокристаллические эпитаксиальные пленки $\mathrm{Fe}_{3} \mathrm{Si}$ и поликристаллические пленки $\mathrm{Fe}_{5} \mathrm{Si}_{3}$ получены на подложках $\mathrm{Si}$ методом молекулярно-лучевой эпитаксии с in situ контролем структуры, оптических и магнитных свойств. Обсуждаются результаты структурных, магнитных и оптических измерений. Экспериментальные данные сравниваются с результатами микроскопического расчета спин-поляризованной электронной структуры, спектров диэлектрической проницаемости и оптической проводимости.

Работа выполнена при поддержке Российского научного фонда (грант № 16-13-00060).

\section{1. Введение}

Наноструктуры на основе кремния - основного материала современной электроники - и ферромагнитных силицидов представляют большой интерес в связи с широким спектром их возможного применения. Эпитаксиальные пленки $\mathrm{Fe}_{3} \mathrm{Si}$ интенсивно исследуются последнее десятилетие с целью создания устройств спинтроники [1-4]. Высокая спиновая поляризация силицида железа $\mathrm{Fe}_{3} \mathrm{Si}$ также открывает возможности для создания ферромагнитных периодических структур, таких как магнитофотонные кристаллы [5]. Кроме того, благодаря их магниторезонансным свойствам [6] эпитаксиальные пленки $\mathrm{Fe}_{3} \mathrm{Si}$ способны найти применение в датчиках слабых магнитных полей и могут использоваться в качестве активного материала в различных электрически управляемых устройствах микроволнового (СВЧ) диапазона. В свою очередь ферромагнитный силицид $\mathrm{Fe}_{5} \mathrm{Si}_{3}$, имеющий температуру Кюри около $390 \mathrm{~K}$, оказывается весьма актуальным для применения в термомагнитных преобразователях энергии [7]. Имеются также свидетельства наличия эффекта гигантского магнетосопротивления, обусловленного формированием наноразмерных частиц $\mathrm{Fe}_{5} \mathrm{Si}_{3}$ в матрице кремния [8]. Первые попытки создания наноструктур $\mathrm{Fe} / \mathrm{Si}$ не привели к успеху из-за формирования металлосилицидных фаз в интерфейсе, которые подавляли спин-поляризованный транспорт в полупроводниковом слое. Рядом исследователей были получены эпитаксиальные пленки различных силицидов, таких как $\mathrm{FeSi}_{2}, \mathrm{FeSi}$ со структурой $\mathrm{CsCl}, \mathrm{Fe}_{3} \mathrm{Si}$ на подложках $\mathrm{Si}$ [9-14]. Исследования эффекта Мессбауэра с изотопическими слоями ${ }^{57} \mathrm{Fe}$ оказались очень полезными для идентификации различных силицидных фаз [15]. Тем не менее полностью силицидных гетероструктур нам неизвестно, в работе [16] сообщается о росте гетероструктуры $\mathrm{Fe}_{3} \mathrm{Si} / \mathrm{Ge} / \mathrm{Fe}_{3} \mathrm{Si} / \mathrm{Si}(111)$. Необходимость введения полупроводникового слоя $\mathrm{Ge}$ вместо $\mathrm{Si}$ обусловлена более низкой температурой эпитаксиального роста германия на $\mathrm{Fe}_{3} \mathrm{Si}$, которую нежелательно поднимать во избежание неконтролируемого роста силицидов в интерфейсе. Дополнительный интерес к металлосилицидам обусловлен их значительными термоэлектрическими свойствами [17]. Поэтому требуются дальнейшие исследования в области технологий создания силицидных гетероструктур и поиска новых силицидных фаз с заданными магнитными и транспортными свойствами. Недавно нами была отработана технология получения монокристаллических слоев ферромагнитного силицида $\mathrm{Fe}_{3} \mathrm{Si}$ на подложках $\mathrm{Si}(111)$ методом молекулярнолучевой эпитаксии [6], а также поликристаллических пленок $\mathrm{Fe}_{5} \mathrm{Si}_{3}$ [18]. В равновесном состоянии фаза $\mathrm{Fe}_{5} \mathrm{Si}_{3}$ существует только при высоких температурах, исключающих интерес к ее возможным магнитным свойствам. В настоящей работе приводятся результаты измерений магнитных свойств и оптических спектров $\mathrm{Fe}_{5} \mathrm{Si}_{3}$ в сравнении с полученными ранее данными для $\mathrm{Fe}_{3} \mathrm{Si}$, a также их сравнение с результатами ab initio расчетов электронной структуры и различных функций отклика, включая спектр оптической проводимости. Нами получена оценка величины статической электропроводности. Знание электронной структуры необходимо для понимания спин-поляризованного транспорта, а также для формирования термоэлектрических свойств.

\section{2. Свойства ферромагнитных пленок $\mathrm{Fe}_{3} \mathrm{Si}$ и $\mathrm{Fe}_{5} \mathrm{Si}_{3}$}

Монокристаллическая пленка $\mathrm{Fe}_{3} \mathrm{Si}$ была получена путем одновременного осаждения железа и кремния на 


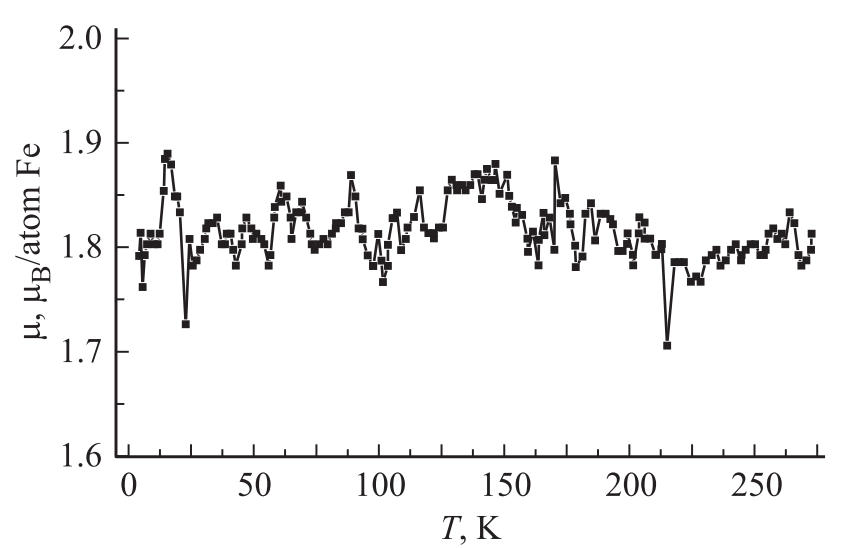

Рис. 1. Температурная зависимость намагниченности $\mathrm{Fe}_{3} \mathrm{Si} / \mathrm{Si}$ в магнитном поле $H=200 \mathrm{Oe}$.

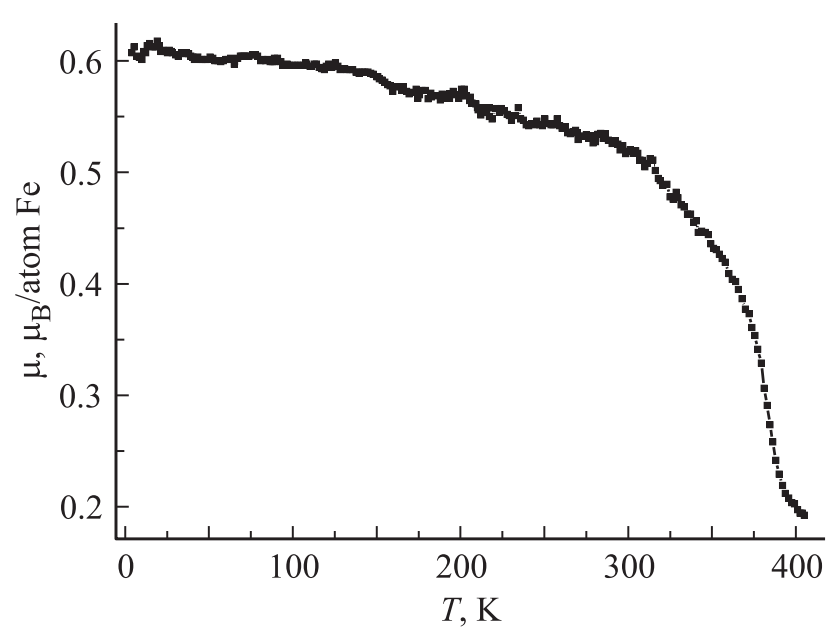

Рис. 2. Температурная зависимость намагниченности $\mathrm{Fe}_{5} \mathrm{Si}_{3}$ в магнитном поле $H=50 \mathrm{Oe}$.

атомарно-чистую поверхность подложки $\mathrm{Si}(111)$ (удельное сопротивление 5-10 $\Omega \cdot \mathrm{cm}$, допирование - бор $\mathrm{B}^{5}$ ) в условиях сверхвысокого вакуума на модернизированной установке молекулярно-лучевой эпитаксии „Ангара“. Базовый вакуум в технологической камере составлял $6.7 \cdot 10^{-8} \mathrm{~Pa}$. Перед синтезом подложка подвергалась химической обработке с последующим термическим отжигом в сверхвысоком вакууме. Одновременное напыление железа и кремния осуществлялось методом термического испарения из двух эффузионных ячеек Кнудсена, изготовленных из высокотемпературного нитрида бора. Скорости осаждения отдельных материалов были откалиброваны методом лазерной эллипсометрии, что обеспечивало стехиометрическое для $\mathrm{Fe}_{3} \mathrm{Si}$ соотношение скоростей потоков $\mathrm{Si}: \mathrm{Fe} \approx 0.57$. Процесс формирования структуры контролировался in situ быстродействующим лазерным эллипсометром ЛЭФ-751M [6] и методом дифракции отраженных быстрых электронов. Интегральная толщина слоя $\mathrm{Fe}_{3} \mathrm{Si}$ по данным спектральной эллипсометрии составила $\sim 27 \mathrm{~nm}$.
Поликристаллическая пленка $\mathrm{Fe}_{5} \mathrm{Si}_{3}$ была получена методом поочередного осаждения субнанометровых слоев $\mathrm{Fe}$ и $\mathrm{Si}$ на поверхность $\mathrm{SiO}_{2} / \mathrm{Si}(100)$ при температуре подложки $330 \mathrm{~K}$ в сверхвысоком вакууме. Поочередное осаждение слоев $\mathrm{Fe}$ и $\mathrm{Si}$ производилось путем термического испарения из эффузионных ячеек Кнудсена. Толщина для каждого слоя железа и кремния была в диапазоне $0.5-0.8 \mathrm{~nm}$. Суммарная толщина структуры $\mathrm{Fe}-\mathrm{Si}$ составила около $27 \mathrm{~nm}$ в пересчете на плотность массивных чистых материалов или $10.7 \pm 0.6 \mu \mathrm{g} / \mathrm{cm}^{2}$ для $\mathrm{Fe}$ и $3.1 \pm 0.1 \mu \mathrm{g} / \mathrm{cm}^{2}$ для Si. После осаждения образец отжигался в сверхвысоком вакууме при температуре $720 \mathrm{~K}$ в течение $30 \mathrm{~min}$ [18]. Рентгеноструктурный анализ образца на порошковом дифрактометре D8 ADVANCE (CuK $K_{\alpha 1,2}$-излучение, Ni-фильтр) с линейным детектором VANTEC показал наличие фазы $\mathrm{Fe}_{5} \mathrm{Si}_{3}$. Следует отметить, что в объемном виде эта фаза существует лишь при высоких температурах и при комнатной температуре термодинамически неустойчива.

Температурная зависимость намагниченности измерялась методом СКВИД-магнитометрии в интервале температур 4-400 K. Для пленки $\mathrm{Fe}_{3} \mathrm{Si}$ намагниченность практически не зависит от температуры в этом диапазоне (рис. 1), что согласуется с величиной температуры Кюри $T_{C}=850 \mathrm{~K}$. Как видно из рис. 2, для $\mathrm{Fe}_{5} \mathrm{Si}_{3}$ температура Кюри много ниже $\left(T_{C} \approx 390 \mathrm{~K}\right)$.

Определение энергетической зависимости оптической проводимости в настоящей работе проводилось на основе данных многоугловой спектральной эллипсометрии с помощью метода, подробно описанного в работе [19]. В расчетах была использована оптическая модель однородной изотропной пленки с неизвестными толщиной и диэлектрической проницаемостью на изотропной подложке $\mathrm{Si}$ с известными оптическими характеристиками. Измерения проводились на быстродействующем спектральном эллипсометре Эллипс-1891 при $T=296$ К.

\section{3. Особенности электронной структуры и оптическая проводимость в $\mathrm{Fe}_{3} \mathrm{Si}$ и $\mathrm{Fe}_{5} \mathrm{Si}_{3}$}

Электронная структура $\mathrm{Fe}_{3} \mathrm{Si}$ была рассчитана в рамках теории функционала плотности в обобщенном градиентном приближении (GGA). Спектр квазичастичных возбуждений был получен как в несамосогласованном $\mathrm{G}_{0} \mathrm{~W}_{0} \quad\left(\mathrm{G}_{0} \mathrm{~W}_{0}\right.$ - несамосогласованный массовый оператор с нулевыми функциями Грина и линией взаимодействия), так и в различных самосогласованных scGW-приближениях (scGW - самосогласованный массовый оператор с „одетыми“ линиями) без учета и с учетом вершинных поправок (в форме из TDDFT теории функционала плотности, зависящего от времени). Во всех вариантах $\mathrm{GW}(\mathrm{GW}$ - массовый оператор в приближении Хедина) в качестве начального шага итераций использовалась зонная структура из 


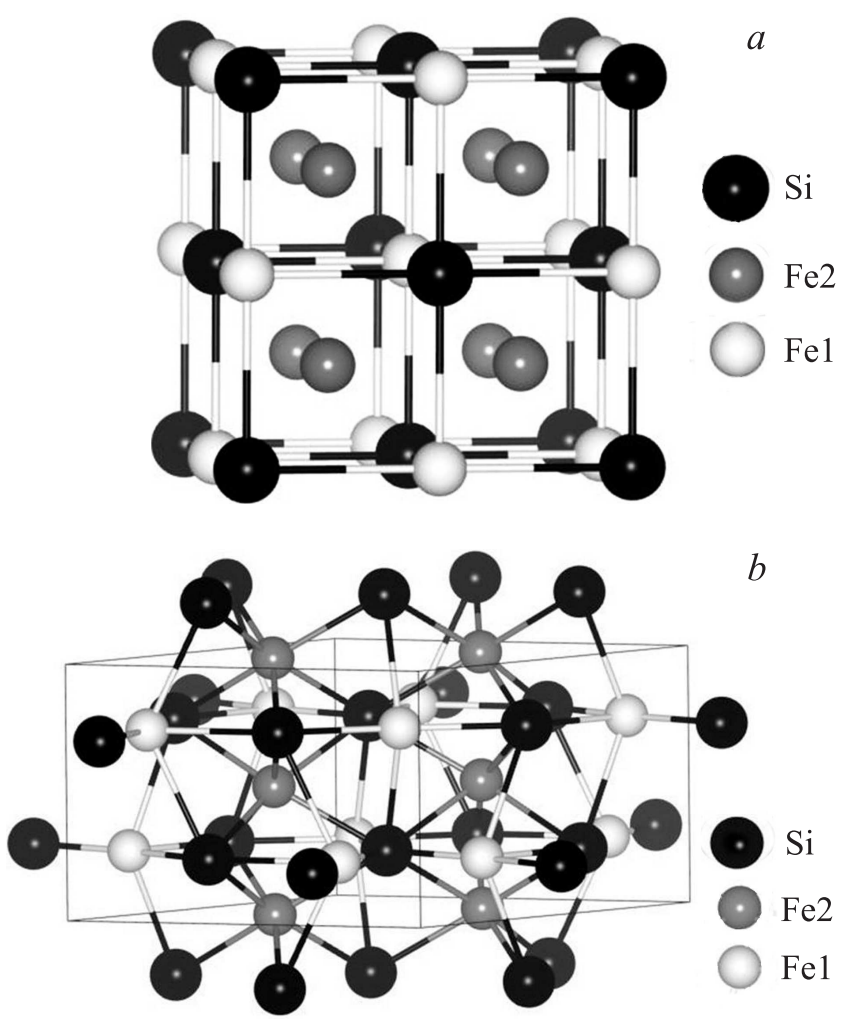

Рис. 3. Структура $\mathrm{Fe}_{3} \mathrm{Si}(a), \mathrm{Fe}_{5} \mathrm{Si}_{3}(b)$ с двумя неэквивалентными ионами железа.
Таблица 1. Заполнение орбиталей для разных позиций и фаз в $\mathrm{Fe}_{3} \mathrm{Si}$

\begin{tabular}{c|c|c|c|c}
\hline \multirow{2}{*}{ Ион } & \multirow{2}{*}{ Орбиталь } & \multicolumn{2}{|c|}{$\mathrm{FM}$} & \multirow{2}{*}{ РМ } \\
\cline { 3 - 4 } & & $\uparrow$ & $\downarrow$ & \\
\hline $\mathrm{Fe} 1$ & $t_{2 g}$ & 0.84 & 0.51 & 0.56 \\
$\left(2.5 \mu_{\mathrm{B}}\right)$ & $e_{g}$ & 0.92 & 0.18 & 0.69 \\
$\mathrm{Fe} 2$ & $t_{2 g}$ & 0.79 & 0.57 & 0.70 \\
$\left(1.5 \mu_{\mathrm{B}}\right)$ & $e_{g}$ & 0.77 & 0.38 & 0.53
\end{tabular}

Приме чание. FM и РM - ферромагнитная и парамагнитная фазы соответственно.

Таблица 2. Заполнение орбиталей для разных позиций и фаз в $\mathrm{Fe}_{5} \mathrm{Si}_{3}$

\begin{tabular}{c|c|c|c|c}
\hline \multirow{2}{*}{ Ион } & \multirow{2}{*}{ Орбиталь } & \multicolumn{2}{|c|}{ FM } & \multirow{2}{*}{ РM } \\
\cline { 3 - 4 } & & $\uparrow$ & $\downarrow$ & \\
\hline $\mathrm{Fe} 1$ & $t_{2 g}(x y)$ & 0.81 & 0.37 & 0.52 \\
$\left(1.9 \mu_{\mathrm{B}}\right)$ & $t_{2 g}(x z)$ & 0.82 & 0.34 & 0.62 \\
& $e_{g}\left(z^{2}\right)$ & 0.80 & 0.46 & 0.62 \\
& $e_{g}\left(x^{2}-y^{2}\right)$ & 0.76 & 0.59 & 0.72 \\
$\mathrm{Fe} 2$ & $t_{2 g}(x y)$ & 0.74 & 0.58 & 0.66 \\
$\left(1.3 \mu_{\mathrm{B}}\right)$ & $t_{2 g}(x z)$ & 0.79 & 0.40 & 0.61 \\
& $e_{g}\left(z^{2}\right)$ & 0.74 & 0.57 & 0.66 \\
& $e_{g}\left(x^{2}-y^{2}\right)$ & 0.73 & 0.57 & 0.65
\end{tabular}
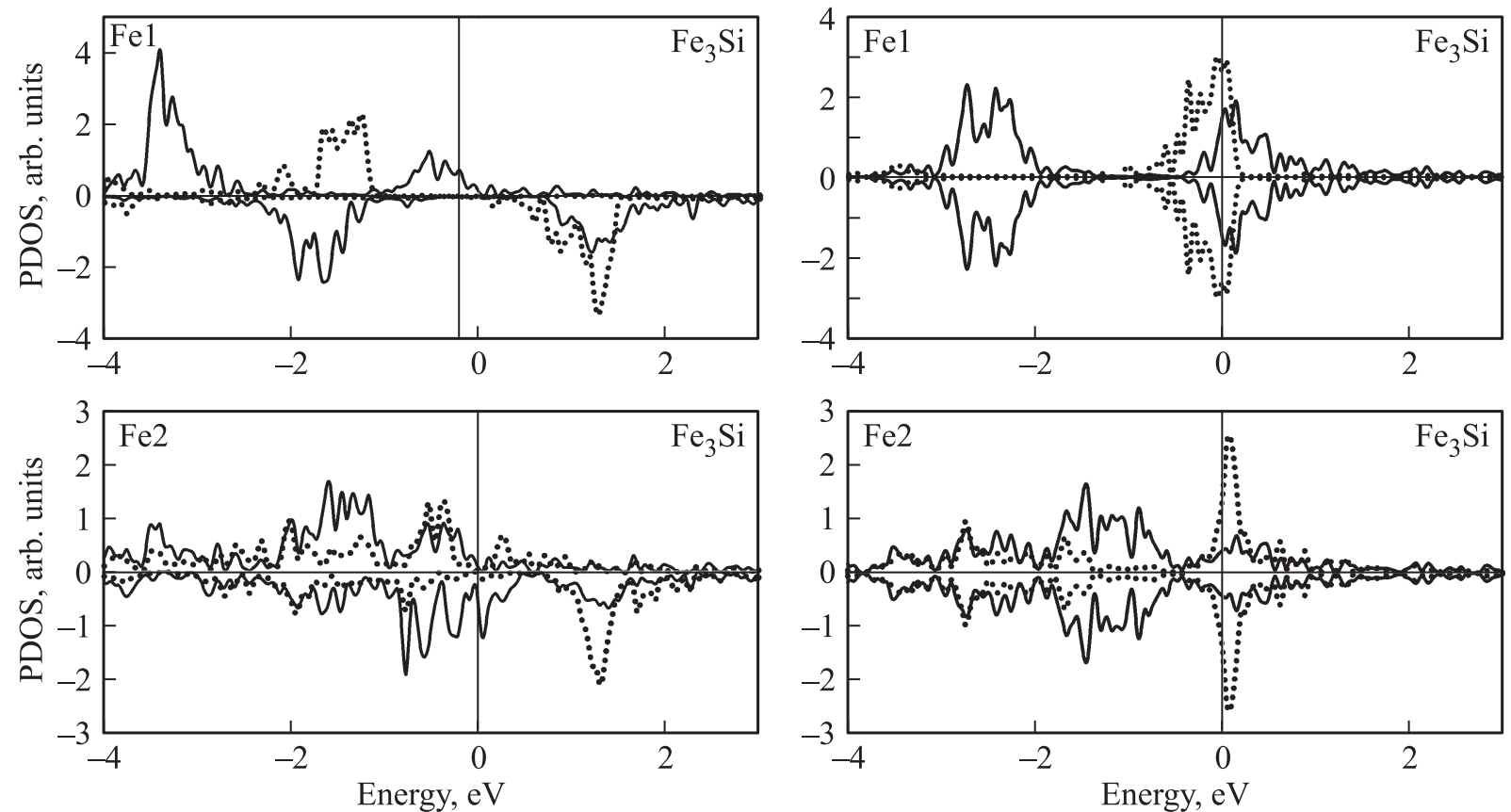

Рис. 4. Парциальные плотности состояний (PDOS) $d$-орбиталей железа $\mathrm{Fe}_{3} \mathrm{Si}$. Сплошная линия $-t_{2 g}$-орбитали, пунктирная $e_{g}$-орбитали. Слева - в ферромагнитной фазе, справа - в парамагнитной фазе (приближение GGA теории функционала плотности). 

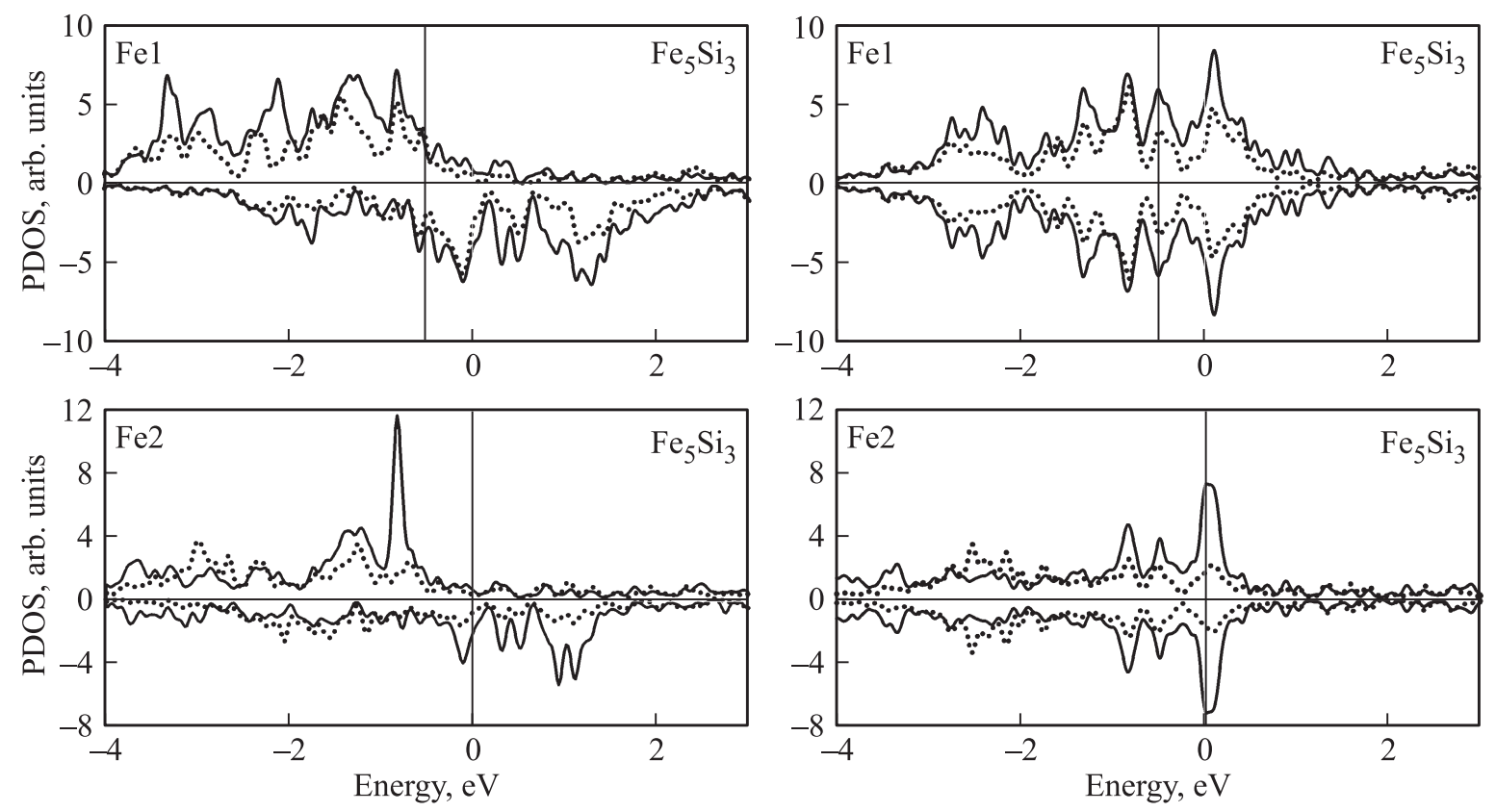

Рис. 5. Парциональные плотности состояний $d$-орбиталей железа $\mathrm{Fe}_{5} \mathrm{Si}_{3}$. Сплошная линия $-t_{2 g}$-орбитали, пунктирная $e_{g}$-орбитали. Слева - в ферромагнитной фазе, справа - в парамагнитной фазе (приближение GGA теории функционала плотности).

GGA-расчета [20]. Спектральные веса электронных возбуждений наиболее сильно меняются в тех областях зоны Бриллюэна, где вклад в формирование зон начинают вносить $d$-электроны. Наиболее хорошо согласуются c экспериментально измеренными на наших образцах спектрами действительной и мнимой частей диэлектрической проницаемости, коэффициентами поглощения и отражения результаты расчетов в scGW-приближении [20]. Магнитное состояние формируется вкладами двух структурно-неэквивалентных ионов железа. Заполнение различных орбиталей для каждой из позиций представлено в табл. 1 и 2.

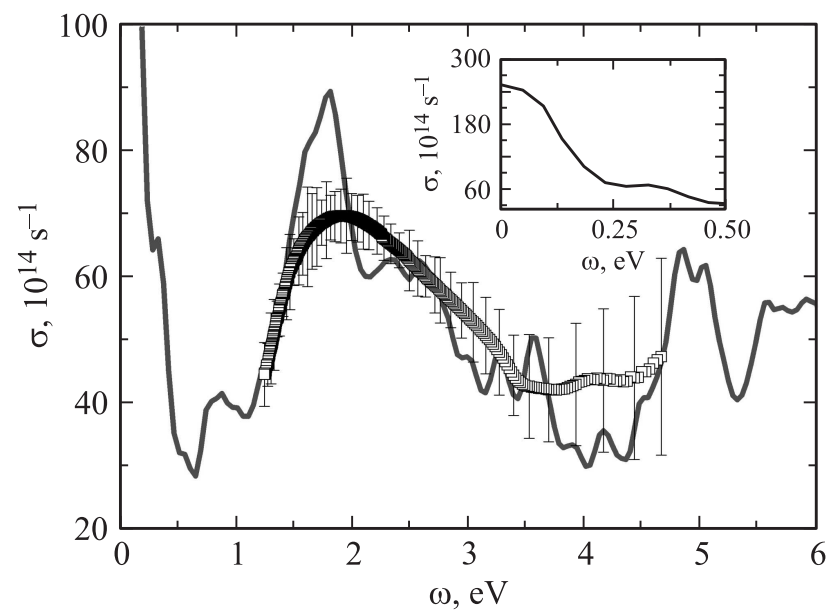

Рис. 6. Оптическая проводимость $\mathrm{Fe}_{3} \mathrm{Si}$ : теоретический расчет в рамках самосогласованного метода scGW (сплошная линия) и экспериментальные результаты (символы). На вставке рассчитанная проводимость при низких частотах.
Ионы Fe1 имеют восемь ближайших соседей $\mathrm{Fe} 2$ в кубическом окружении и магнитный момент $M_{1}=2.52 \mu_{\mathrm{B}}$ (рис. 3). Ионы $\mathrm{Fe} 2$ находятся в тетраэдрическом окружении из ионов $\mathrm{Fe} 1$ и $\mathrm{Si}$, их магнитный момент равен $M_{2}=1.34 \mu_{\mathrm{B}}$. На рис 4 представлены плотности состояний $\mathrm{Fe}_{3} \mathrm{Si}$ в ферромагнитной и парамагнитной фазах отдельно для каждой позиции железа, а на рис. 5 - то же самое для $\mathrm{Fe}_{5} \mathrm{Si}_{3}$.

Сравнение зонных структур обоих силицидов в ферромагнитном и парамагнитном состояниях показывает, что трансформация зонной структуры при магнитном упорядочении происходит более сложно, чем в простой модели Стонера. В то же время для обоих силицидов в парамагнитном состоянии имеется пик в плотности состояний на уровне Ферми, способствующий выполнению критерия Стонера.

\section{4. Обсуждение результатов}

Для сравнения рассчитанных и измеренных экспериментально электронных свойств $\mathrm{Fe}_{3} \mathrm{Si}$ мы приводим на рис. 6 спектры оптической проводимости. Видно, что есть качественное согласие с экспериментом при частотах, доступных в наших измерениях. В этом диапазоне частот доминирующий вклад в проводимость вносят межзонные переходы. При учете внутризонных переходов низкочастотная расходимость обрезается величиной статической проводимости. В рамках зонных расчетов возможна оценка статической проводимости в модели Друде-Лоренца $\sigma_{0}=\left(\omega_{p}^{2} \tau\right) / 4 \pi$, в которую входят вычисленные из первых принципов параметры 
электронной структуры - плазменная частота $\omega_{p}-$ и один феноменологический параметр - время релаксации $\tau$. При типичном для $3 d$-металлов значении времени релаксации $\tau=10 \mathrm{fs}$ [21] мы получили для $\mathrm{Fe}_{3} \mathrm{Si}$ величину статической проводимости $\sigma_{0}=2.5 \cdot 10^{6} \mathrm{~S} / \mathrm{m}$. Измеренная на наших образцах проводимость при температуре $6.7 \mathrm{~K}$ оказалась равной $\sigma_{0}=1.82 \cdot 10^{6} \mathrm{~S} / \mathrm{m}$. Сравнивая с проводимостью чистого железа $10^{7} \mathrm{~S} / \mathrm{m}$, видим, что у силицида $\mathrm{Fe}_{3} \mathrm{Si}$ проводимость меньше, чем у чистых металлов, хотя он и является металлическим соединением. Намного меньшее значение температуры Кюри $(390 \mathrm{~K})$ для фазы $\mathrm{Fe}_{5} \mathrm{Si}_{3}$ может обусловливать более сильные спиновые флуктуации и их влияние на проводимость и термоэлектрические свойства в области температур $T \sim 300-500 \mathrm{~K}$ по сравнению с имеющими место для $\mathrm{Fe}_{3} \mathrm{Si}$.

Согласно формуле Иоффе для термоэлектрической добротности, высококачественный термоэлектрический материал должен одновременно иметь высокую электропроводность, большую термоэдс и низкую теплопроводность. Металлы имеют высокую проводимость, но большую теплопроводность и низкую термоэдс. Влияние пространственной неоднородности в наших наноструктурах дает основание надеяться на уменьшение теплопроводности. Термоэлектрические свойства созданных наноструктур являются предметом экспериментального исследования в настоящее время.

Авторы выражают глубокую благодарность И.С. Сандалову за плодотворное обсуждение и помощь в интерпретации полученных результатов.

\section{Список литературы}

[1] H.Y. Hung, S.Y. Huang, P. Chang, W.C. Lin, Y.C. Liu, S.F. Lee, M. Hong, J. Kwo. J. Cryst. Growth. 323, 372 (2011).

[2] T. Yoshitake, D. Nakagauchi, T. Ogawa, M. Itakura, N. Kuwano, Y. Tomokiyo, T. Kajiwara, K. Nagayama. Appl. Phys. Lett. 86, 262505 (2005).

[3] Y. Ando, K. Hamaya, K. Kasahara, Y. Kishi, K. Ueda, K. Sawano, T. Sadoh, M. Miyao. Appl. Phys. Lett. 94, 182105 (2009).

[4] Y. Fujita, S. Yamada, Y. Ando, K. Sawano, H. Itoh, M. Miyao, K. Hamaya. J. Appl. Phys. 113, 013916 (2013).

[5] Y. Maeda, T. Ikeda, T. Ichikawa, T. Nakajima, B. Matsukura, T. Sadoh, M. Miyao. Phys. Procedia 11, 200 (2011).

[6] И.А. Яковлев, С.Н. Варнаков, Б.А. Беляев, С.М. Жарков, М.С. Молокеев, И.А. Тарасов, С.Г. Овчинников. Письма в ЖЭТФ 99, 610 (2014).

[7] A. Post, C. Knight, E. Kisi. J. Appl. Phys. 114, 033915 (2013).

[8] K.S.K. Varadwaj, K. Seo, J. In, P. Mohanty, J. Park, B. Kim. Am. Chem. Soc. 129, 8594 (2007).

[9] H. von Kanel, R. Stalder, H. Sirringhaus, N. Onda, J. Henz. Appl. Surf. Sci. 53, 196 (1991).

[10] H. von Kanel, K.A. Mader, E. Muller, N. Onda, H. Sirringhaus. Phys. Rev. B 45, 13807 (1992).

[11] E.V. Chubunova, I.D. Khabelashvili, Y.Y. Lebedinskii, V.N. Nevolin, A. Zenkevich. Thin Solid Films 247, 39 (1994).
[12] G.J. Strijkers, J.T. Kohlhepp, H.J.M. Swagten, W.J.M. de Jonge. Phys. Rev. B 60, 9583 (1999).

[13] M.V. Gomoyunova, D.E. Malygin, I.I. Pronin, A.S. Voronchikhin, D.V. Vyalikh, S.L. Molodtsov. Surf. Sci. 601, 5069 (2007).

[14] H.F. Hsu, H.Y. Wu, Y.T. Huang, T.H. Chen. Jpn. J. Appl. Phys. 48, 08JB09 (2009).

[15] M. Fanciulli, G. Weyer, H. von Kanel, N. Onda. Phys. Scripta T 54, 16 (1994).

[16] S. Yamada, M. Miyao, K. Hamaya. In: Proc. of 7th Int. SiliconGermanium Technology and Device Meeting (ISTDM). Singapore (2014). P. 55.

[17] Thermoelectrics handbook: macro to nano / Ed. D.M. Rowe. CRC Press, Taylor \& Francis Group (2006). 954 p.

[18] С.А. Лященко, З.И. Попов, С.Н. Варнаков, Е.А. Попов, М.С. Молокеев, И.А. Яковлев, А.А. Кузубов, С.Г. Овчинников. ЖЭТФ 147, 1023 (2015).

[19] F.K. Urban III, D. Barton, T. Tiwald. Thin Solid Films 518, 1411 (2009).

[20] I. Sandalov, N. Zamkova, V. Zhandun, I. Tarasov, S. Varnakov, I. Yakovlev, L. Solovyov, S. Ovchinnikov. Phys. Rev. B 92, 205129 (2015).

[21] Н. Ашкрофт, Н. Мермин. Физика твердого тела. Мир, М. (1979). 795 c.

Публикация материалов Симпозиума завершена. 